\title{
La complejidad terapéutica tras el implante de una válvula aórtica transcatéter (TAVI): un caso infrecuente de trombosis y endocarditis
}

\author{
The complex management after transcatheter aortic valve implantation (TAVI): \\ $A$ rare case of thrombosis and endocarditis
}

\author{
Paula Morlanes-Gracia*, Jorge Melero-Polo, Adrián Riaño-Ondiviela, Ana Martínez-Labuena, Sara C. \\ Río-Sánchez y Daniel Meseguer-González
}

Servicio de Cardiología, Hospital Clínico Universitario Lozano Blesa. Zaragoza, España

La implantación de una válvula aórtica transcatéter (TAVI, transcatheter aortic valve implantation) es una práctica extendida en pacientes con estenosis aórtica de riesgo quirúrgico intermedio y alto. Se han descrito complicaciones como endocarditis e incluso trombosis valvular clínica, aunque con una incidencia de esta última de tan sólo $0.6 \%$ a $2.8 \%$ y es más frecuente luego los procedimientos valve-in-valve y válvulas expandibles con balón ${ }^{1}$. La trombosis debe sospecharse en cualquier paciente con síntomas recientes de insuficiencia cardíaca, un episodio embólico o aumento manifiesto de los gradientes. La antivitamina $\mathrm{K}$ o la heparina no fraccionada son la primera línea de tratamiento $^{2}$. En la actualidad existe controversia acerca del tratamiento y la necesidad de la doble antiagregación tras el implante, ya que ésta parece aumentar los episodios hemorrágicos sin reducir las complicaciones isquémicas ${ }^{3}$.

Se presenta el caso de un paciente varón de 85 años con antecedentes de fibrilación auricular paroxística y estenosis aórtica grave elegible para TAVI. En la coronariografía diagnóstica se realizó intervencionismo percutáneo con implante de tres stents farmacoactivos sobre una lesión grave en la arteria coronaria derecha. Dos meses antes del ingreso se intervino la estenosis aórtica grave mediante la implantación del TAVI SAPIEN 3 ULTRA (Edwars Lifesciences; Irvine, California, EE.UU.) de $26 \mathrm{~mm}$ con buen resultado (gradientes medio de $15 \mathrm{mmHg}$ y máximo de $26 \mathrm{mmHg}$ después de la intervención). Recibió el alta con ácido acetilsalicílico (100 mg), clopidogrel (75 mg/24 h) y apixabán (5 mg/24 h).

Acude a urgencias por disnea de esfuerzo, ortopnea progresiva y bendopnea. El paciente negó posibles desencadenantes, como clínica infecciosa, mal cumplimiento terapéutico o transgresiones dietéticas. En la exploración se identificó un soplo sistólico aórtico rudo. Los estudios detectaron valores de NT-proBNP elevados de $7,610 \mathrm{pg} / \mathrm{ml}$ y el ecocardiograma al pie de la cama documentó un incremento significativo del gradiente transprotésico (gradiente medio de $52 \mathrm{mmHg}$ y máximo de $81 \mathrm{mmHg}$ ). Por todo lo anterior ingresó al servicio de cardiología con sospecha de trombosis subguda de la prótesis valvular.

Se extrajeron hemocultivos en frío a pesar de la ausencia de datos infecciosos y, ante la sospecha de una
Correspondencia:

*Paula Morlanes-Gracia

E-mail: Pmg89@live.com

1405-9940 / ( 2021 Instituto Nacional de Cardiología Ignacio Chávez. Publicado por Permanyer. Este es un artículo open access bajo la licencia CC BY-NC-ND (http://creativecommons.org/licenses/by-nc-nd/4.0/).
Disponible en internet: 02-06-2021 Arch Cardiol Mex. 2021;91(4):519-521 www.archivoscardiologia.com 
posible trombosis protésica, se solicitó angio-TC torácica, en la que se identificó imagen hipodensa de $16.7 \mathrm{~mm}$ en contacto con el seno coronario derecho de unas 70 unidades Hounsfield, indicativa de un trombo a ese nivel (Fig. 1). Se inició perfusión de heparina sódica y a las 24 horas de ingreso desarrolló dolor y parestesias en la extremidad inferior izquierda, con frialdad, palidez y ausencia de pulso. Se realizó ecografía Doppler en dicha extremidad que confirmó isquemia arterial aguda de origen embólico y la paciente se sometió a embolectomía transfemoral izquierda, que extrajo el émbolo completo de $14 \mathrm{~mm}$.

Un nuevo ecocardiograma reveló una reducción de los gradientes, pero se identificó un aislamiento de Streptococcus salivarius en cuatro hemocultivos. Se instituyó tratamiento con ceftriaxona y gentamicina y se mantuvo el tratamiento con heparina sódica. Ante el episodio embólico y endocarditis probable se llevó el caso al equipo de cardiología y se decidió indicar un tratamiento conservador por el elevado riesgo quirúrgico del paciente y el rechazo de éste a otros tratamientos invasivos.

En los hemocultivos de control no se detectó crecimiento de ningún germen. Sin embargo, durante los días posteriores se identificó un tiempo de tromboplastina parcial activado de difícil control con tendencia a los límites infraterapéuticos; se realizó un ecocardiograma transesofágico a los siete días de ingreso que mostró imágenes de una vegetación de $11 \times 7.5 \mathrm{~mm}$, así como nuevo aumento del gradiente transprotésico (gradiente máximo de $70 \mathrm{mmHg}$ ) (Fig. 2). Dada la coincidencia del nuevo incremento de los gradientes con mal control del tratamiento anticoagulante, y puesto que los hemocultivos se habían tornado negativos con anterioridad, se consideró una nueva trombosis y se decidió comenzar tratamiento con acenocumarol. Al final se repitió el estudio transesofágico y se observó la desaparición de la imagen indicativa de trombo/vegetación y la normalización del gradiente transprotésico. Sin embargo, 48 horas más tarde el paciente mostró focalidad neurológica; se solicitó TAC cerebral urgente que demostró una hemorragia intracraneal masiva con desplazamiento de la línea media y consecuente fallecimiento del paciente.

Los criterios de Latib, et al. para la definición de trombosis protésica ya se han utilizado en estudios observacionales y son los siguientes: a) disfunción valvular (gradiente medio $>20 \mathrm{mmHg}$, reducción del área valvular $<1.2 \mathrm{~cm}^{2}$ o regurgitación transvalvular más que ligera de nueva aparición) secundaria a trombosis diagnosticada con un método de imagen (ecografía o

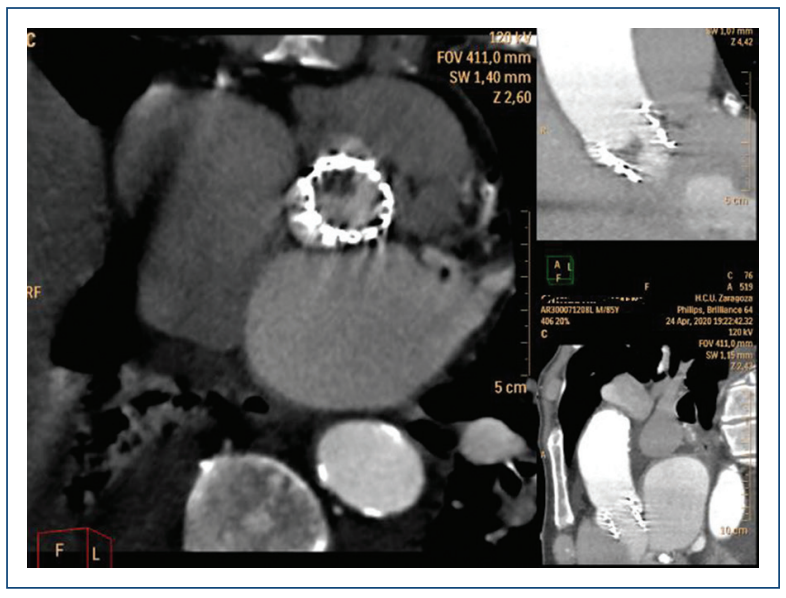

Figura 1. Hipodensidad indicativa de trombo en la TAC torácica.

tomografía computarizada multidetector) o por respuesta al tratamiento anticoagulante; b) masa móvil sospechosa de trombo en la válvula sin considerar disfunción y en ausencia de infección ${ }^{1}$. La trombosis subclínica de una válvula protésica de implante percutáneo se ha descrito en un $3 \%$ a $4 \%$ de los pacientes portadores de prótesis valvulares expandibles con balón transcurrido el primer mes luego de la intervención y también en otros tipos de TAVI y prótesis valvulares biológicas implantadas de forma quirúrgica. Sin embargo, la trombosis clínica, como se presenta en este caso, es más infrecuente: en algunas series se ha descrito en $<1 \%{ }^{1,4}$. El tratamiento antitrombótico tras el implante del TAVI es todavía un reto. Las guías recomiendan el uso de clopidogrel más ácido acetilsalicílico durante tres a seis meses después del TAVI. Sin embargo, no es claro el beneficio de la doble antiagregación en estos pacientes. En el reciente estudio Popular $T A V I$, la antiagregación simple con ácido acetilsalicílico se relacionó con menor incidencia de hemorragias y mostró ser no inferior al ácido acetilsalicílico más clopidogrel para el combinado de episodios trombóticos ${ }^{3}$. No obstante, su recomendación es clara si se ha realizado además revascularización coronaria percutánea. No se conoce la combinación óptima de inhibidores plaquetarios para los pacientes que necesitan anticoagulación después de TAVI ni en otros escenarios como el tratamiento en los pacientes intervenidos de TAVI y enfermedad coronaria con indicación previa de anticoagulación.

La incidencia de endocarditis infecciosa en pacientes sometidos a TAVI es de $5 \%$ a $8 \%$ a 5 años y el primer año es el período de mayor riesgo ${ }^{5}$. En este caso se 


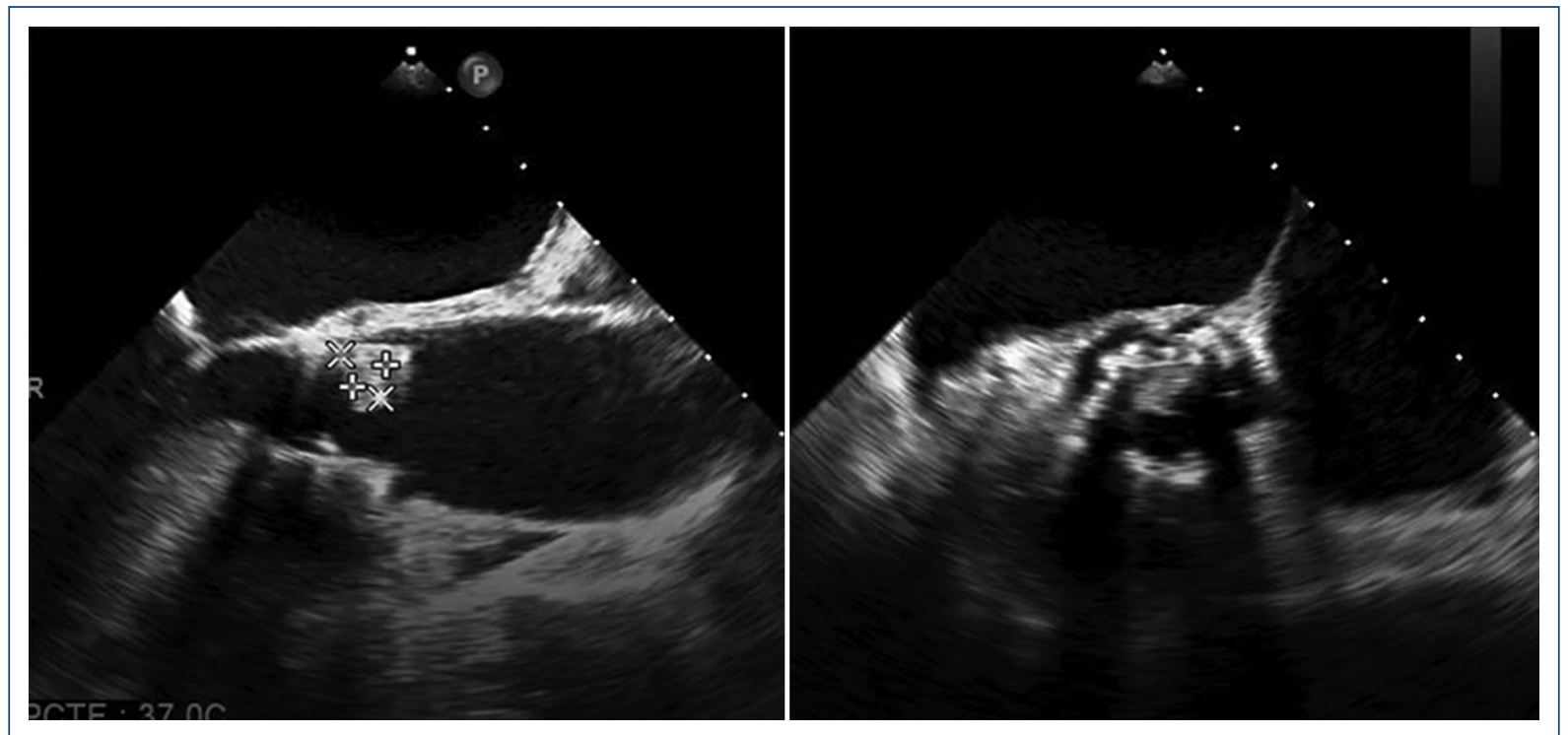

Figura 2. Vegetación sobre la válvula aórtica protésica.

aisló Streptococcus salivarius en hemocultivos, que constituye un criterio menor, y no se realizó TEP-TAC por el implante de TAVI reciente que implica una respuesta inflamatoria. No obstante, se inició antibioticoterapia y se intentó optimizar el tratamiento anticoagulante que destacó por su difícil control, incluido un efecto adverso hemorrágico de mal pronóstico; esto revela el difícil control que supone algunas veces esta complicación.

\section{Financiamiento}

Ninguno.

\section{Conflicto de intereses}

Ninguno.

\section{Responsabilidades éticas}

Protección de personas y animales. Los autores declaran que para esta investigación no se han realizado experimentos en seres humanos ni en animales.

Confidencialidad de los datos. Los autores declaran que han seguido los protocolos de su centro de trabajo sobre la publicación de datos de pacientes.

Derecho a la privacidad y consentimiento informado. Los autores han obtenido el consentimiento informado de los pacientes o sujetos referidos en el artículo. Este documento obra en poder del autor de correspondencia.

\section{Bibliografía}

1. Jose J, Sulimov D, El-Mawardy M, Sato T, Allali A, Holy EW, et al. Clinical bioprosthetic heart valve thrombosis after transcatheter aortic valve replacement. Incidence, characteristics and treatment outcomes JACC Cardivasc Interv. 2017;10(7):686-97.

2. Habib G, Lancellotti $P$, Antunes MJ, Bongiorni MG, Casalta JP, Zotti F et al. Guía ESC 2015 sobre el tratamiento de la endocarditis infecciosa. Rev Esp Cardiol. 2016;69(1):69.e1-e49.

3. Brouwer J, Nijenhuis VJ, Delewi R, Hermanides RS, Holvoet W Dubois $\mathrm{C}$, et al. Aspirin with or without clopidogrel after transcatheter aortic-valve implantation. N Engl J Med. 2020;383:1447-1457.

4. Moral S, Carballo J, Ballesteros E, Panaro A, Evangelista A. Trombosis valvular "subclínica" tras implante percutáneo de válvula aórtica: ¿un riesgo latente? Rev Esp Cardiol. 2016;70:512-514.

5. Butt JH, Ihlemann N, De Backer O, Sondergaard L, Havers-Borgersen E, Gislason $\mathrm{GH}$, et al. Long-term risk of infective endocarditis after transcatheter aortic valve replacement. J Am Coll Cardiol. 2019;73:1646-55. 